\title{
Evaluation of protective efficacy of three novel H3N2 canine influenza vaccines
}

\author{
Liqing Tu' ${ }^{1,2,3, *}$, Pei Zhou ${ }^{1,2,3, *}$, Lutao Li ${ }^{1,2,3}$, Xiuzhen $\mathrm{Li}^{1}$, Renjun $\mathrm{Hu}^{1,2,3}$, Kun Jia ${ }^{1,2,3}$, \\ Lingshuang Sun ${ }^{1,2,3}$, Ziguo Yuan ${ }^{1}$ and Shoujun Li ${ }^{1,2,3}$ \\ ${ }^{1}$ College of Veterinary Medicine, South China Agricultural University, Guangzhou, Guangdong Province 510642, People's \\ Republic of China \\ ${ }^{2}$ Guangdong Provincial Key Laboratory of Prevention and Control for Severe Clinical Animal Diseases, Guangzhou, Guangdong \\ Province 510642, People's Republic of China \\ ${ }^{3}$ Guangdong Provincial Pet Engineering Technology Research Center, Guangzhou, Guangdong Province 510642, People's \\ Republic of China \\ *These authors contributed equally to this work
}

Correspondence to: Shoujun Li, email: shoujunli@scau.edu.cn Ziguo Yuan, email: ziguoyuan@scau.edu.cn

Keywords: canine influenza vaccine, H3N2, pVAX1-HA, rCAV2-HA, inactivated CIV

Received: July 12, $2017 \quad$ Accepted: August 29, $2017 \quad$ Published: September 20, 2017

Copyright: Tu et al. This is an open-access article distributed under the terms of the Creative Commons Attribution License 3.0 (CC BY $3.0)$, which permits unrestricted use, distribution, and reproduction in any medium, provided the original author and source are credited.

\section{ABSTRACT}

Canine influenza virus (CIV) has the potential risk to spread in different areas and dog types. Thus, there is a growing need to develop an effective vaccine to control CIV disease. Here, we developed three vaccine candidates: 1) a recombinant pVAX1 vector expressing H3N2 CIV hemagglutinin (PVAX1-HA); 2) a live attenuated canine adenovirus type 2 expressing H3N2 CIV hemagglutinin (rCAV2-HA); and 3) an inactivated H3N2 CIV (A/canine/Guangdong/01/2006 (H3N2)). Mice received an initial intramuscular immunization that followed two booster injections at 2 and 4 weeks post-vaccination (wpv). The splenic lymphocytes were collected to assess the immune responses at 6 wpv. The protective efficacy was evaluated by challenging H3N2 CIV after vaccination (at $6 \mathrm{wpv}$ ). Our results demonstrated that all three vaccine candidates elicited cytokine and antibody responses in mice. The rCAV2-HA vaccine and the inactivated vaccine generated efficient protective efficacy in mice, whereas limited protection was provided by the pVAX1-HA DNA vaccine. Therefore, both the rCAV2-HA live recombinant virus and the inactivated CIV could be used as potential novel vaccines against H3N2CIV. This study provides guidance for choosing the most appropriate vaccine for the prevention and control of CIV disease.

\section{INTRODUCTION}

In 2004, canine influenza virus (CIV) was first reported in Florida (United States of America, USA) [1]. CIV originated from the equine influenza virus (EIV) H3N8. However, H3N2 CIV of avian origin was subsequently identified in 2007 among dogs in South Korea [2] and was further traced back to 2006 when it was identified in China according to our previous study [3]. In 2015, a H3N2 CIV outbreak occurred in Chicago, and the virus then rapidly spread to numerous states in the USA $[4,5]$. And, seroepidemiological evidence of H3N8
CIV among pet dogs was reported in China according to our previous study [6].

$\mathrm{CIV}$, mainly including $\mathrm{H} 3 \mathrm{~N} 8[1,7,8]$ and $\mathrm{H} 3 \mathrm{~N} 2$ $[2,9]$, naturally or artificially infects numerous species, such as horses [10], cats [11], mice [12], ferrets [13], and pigs [14]. Given that CIV represents a potential risk for interspecies and crosspieces transmission [15], there is a growing concern regarding the epidemic threat that CIV poses. Indeed, the number of cases of natural H3N2 CIV infection in dogs has been increasing yearly $[9,16,17]$, increasing the risk of viral exposure to animal populations or humans via infected animals. Therefore, the prevention 
and control of CIV disease is necessary for animal health and public health concerns.

Over recent decades, influenza A virus vaccines have been developed to contain several viral strains, and different vector types have been used for delivery. The most common human influenza A vaccine contains an inactivated virus [18-20]. Furthermore, multiple antigenic peptidebased vaccines [21-23], live attenuated influenza virus [24-27], recombined adenovirus [28-30], and DNA vaccines $[31,32]$ that express one or more viral antigens [33] have demonstrated protective efficacy against influenza.

In an attempt to develop a vaccine against CIV, a canarypox-vectored EIV H3N8 vaccine expressing hemagglutinins of A/equine/Kentucky/94 (vCP1529) and A2/equine/Ohio /03 (vCP2242) and an equine herpesvirus type 1 recombinant EIV $\mathrm{H} 3 \mathrm{~N} 8$ vaccine expressing hemagglutinins of A/canine/PA/10915-07 were evaluated against $\mathrm{H} 3 \mathrm{~N} 8 \mathrm{CIV}$ in dogs $[34,35]$. In addition, the inactivated H3N2 CIV vaccine (A/canine/Korea/02/07) provides protection against $\mathrm{H} 3 \mathrm{~N} 2 \mathrm{CIV}$ infection in dogs [36]. However, only commercial inactivated H3N8 CIV and $\mathrm{H} 3 \mathrm{~N} 2 \mathrm{CIV}$ vaccines are approved in the USA [37]. In this study, we generated and evaluated three H3N2 CIV vaccine candidates, including a recombinant DNA vaccine, recombinant canine adenovirus, and an inactivated CIV vaccine. Here, we assessed the immunogenicity and protective efficacy elicited by each vaccine in mice. This study provides guidance for choosing the most appropriate vaccine platform for the prevention and control of $\mathrm{H} 3 \mathrm{~N} 2$ CIV disease.

\section{RESULTS}

\section{CIV vaccine candidates were successfully prepared}

Figure 1A demonstrates that the HA gene from CIV (A/canine/Guangdong/01/2006 (H3N2)) was favorably inserted into the pVAX1 and ppoly2-CAV2- $\Delta \mathrm{E}$ (the partial E3 region of canine adenovirus type 2) vectors. The recombined pVAX1-HA vector was transferred into Madin-Darby canine kidney (MDCK) cells for $72 \mathrm{~h}$. HA expression from the pVAX1-HA vector was detected by an indirect immunofluorescence test (Figure 1B) and PCR analysis of MDCK cells (data not shown). In addition, MDCK cells elicited the typical cytopathic effect of an adenovirus infection after infection with rCAV2-HA (recombined CAV2-HA virus) for $24 \mathrm{~h}$ (Figure 1C), as characterized by the grape-like gathering of cells adjacent to the infected cells. HA expression by rCAV2-HA was verified by Western blot (Figure 1D).

\section{HI assay}

At two, four, six, and eight weeks after the initial vaccination, serum samples were collected for antibody assessment. Samples from mice vaccinated with pVAX1HA, rCAV2-HA, and an inactivated CIV vaccine all exhibited an antibody response against CIV as defined by a hemagglutination inhibition (HI) titer $\geq 2^{4}$ (Table 1). As expected, the HI titers of the control groups vaccinated with CAV2, pVAX1 vector, adjuvant alone, or phosphate buffered solution (PBS) were negative $(<1: 2)$ (Table 1$)$.

\section{Lymphocyte proliferation and cytokine levels}

Splenic lymphocyte proliferation in mice $(n=3$ / group) was detected using a CCK-8 kit at 6 wpv. The stimulation index (SI) revealed prominent increases in lymphocytes in mice vaccinated with pVAX1-HA, rCAV2$\mathrm{HA}$, and inactivated vaccine (Figure $2 \mathrm{~A}$ ). Compared with the negative control, the SI of the splenic lymphocytes of mice vaccinated with the pVAX1-HA DNA vaccine, the live rCAV2-HA and the inactivated vaccine were significantly increased to 3.49-, 6.03- and 5.81-fold, respectively.

In addition, splenic lymphocyte cultures were collected to quantify the level of cytokine production after HA protein antigen stimulation for $72 \mathrm{~h}$. All cytokine levels (interleukin (IL)-2, IL-4, IL-10 and interferon (IFN)- $\gamma$ ) in mice vaccinated with pVAX1-HA, rCAV2$\mathrm{HA}$, and inactivated CIV were significantly increased compared with mice vaccinated with empty plasmid, adjuvant or PBS $(p<0.05)$ (Figure 2B).

\section{All CIV vaccines exhibit protective efficacy against CIV infection}

The rectal temperature of mice was measured every day post-challenge. However, a relatively constant temperature of $36.8 \pm 0.2^{\circ} \mathrm{C}$ was maintained (data not shown). At 5 days post-challenge (dpc), lungs were collected for pathology. Gross lung lesions (hemorrhages and tumidness) were characterized in all control groups (Figure 3A). The lungs also exhibited severe and extensive histopathologic changes (hematoxylin and eosin (HE) stain) (Figure 3B). Specifically, the alveolar septa were thickened, and the alveolar lumen was infiltrated with neutrophils and other inflammatory cells (Figure 3A. I IV. In addition, lung sections from challenged mice vaccinated with pVAX1-HA exhibited mild pathology with only a moderate thickening of the alveolar septa in some instances (Figure 3B.VII). Lung lesions in mice vaccinated with rCAV2-HA exhibited moderate pathology (Figure 3B.VI) that included a partial bronchus filled with small neutrophils. Remarkably, the lungs of the group vaccinated with inactivated vaccine (Figure $3 \mathrm{AV}$ ) exhibited no difference compared with the unchallenged control group (Figure 3A.IX).

In Figure 4A, the virus titers of lungs and tracheas from vaccinate mice were significantly reduced compared with control mice, suggesting that all candidate vaccines 
were protective against virus replication in the respiratory tract (Figure 4A).

The percent of weight change was based on the starting weight on $0 \mathrm{dpc}$. In this trial body weight loss was not noted in mice from the rCAV2 and inactivated groups, but slight body weight loss was noted in mice from the pVAX1-HA DNA group. However, significant body weight loss was noted in mice in the no vaccination control groups (pVAX1 group, CAV2 group, adjuvant group, NI group and PBS group), and the body weight loss rate exhibited its lowest point on $6 \mathrm{dpc}$ (Figure 4B).

\section{DISCUSSION}

A hallmark of the influenza virus is the remarkable variability of its major surface glycoproteins. HA is one such surface glycoprotein that has been used in recombinant vaccines against influenza because it is a dominant antigen that can enhance the immune response to influenza [38]. The HA amino acid sequence of the A/ canine/Guangdong/01/2006 (H3N2) virus used in this study was $97.2 \% \sim 98.9 \%$ similar to 16 other H3N2 CIV strains isolated in China and Korea, indicating that this strain has the potential to be a universal vaccine to against H3N2 CIVs. Mice have been used as a traditional animal model for influenza virus infection and vaccination [39]. In this study, the immunogenicity and protective efficacy were analyzed by vaccinating mice with three types of CIV vaccines, including a pVAX1-HA DNA vaccine, a live rCAV2-HA vaccine, and an inactivated vaccine.

As previously reported, the plasmid pVAX1 serves as a promising approach for antigen preparation in vaccine development. This DNA vaccine vector system is ideal for high-level recombinant protein production in $\mathrm{CHO}$
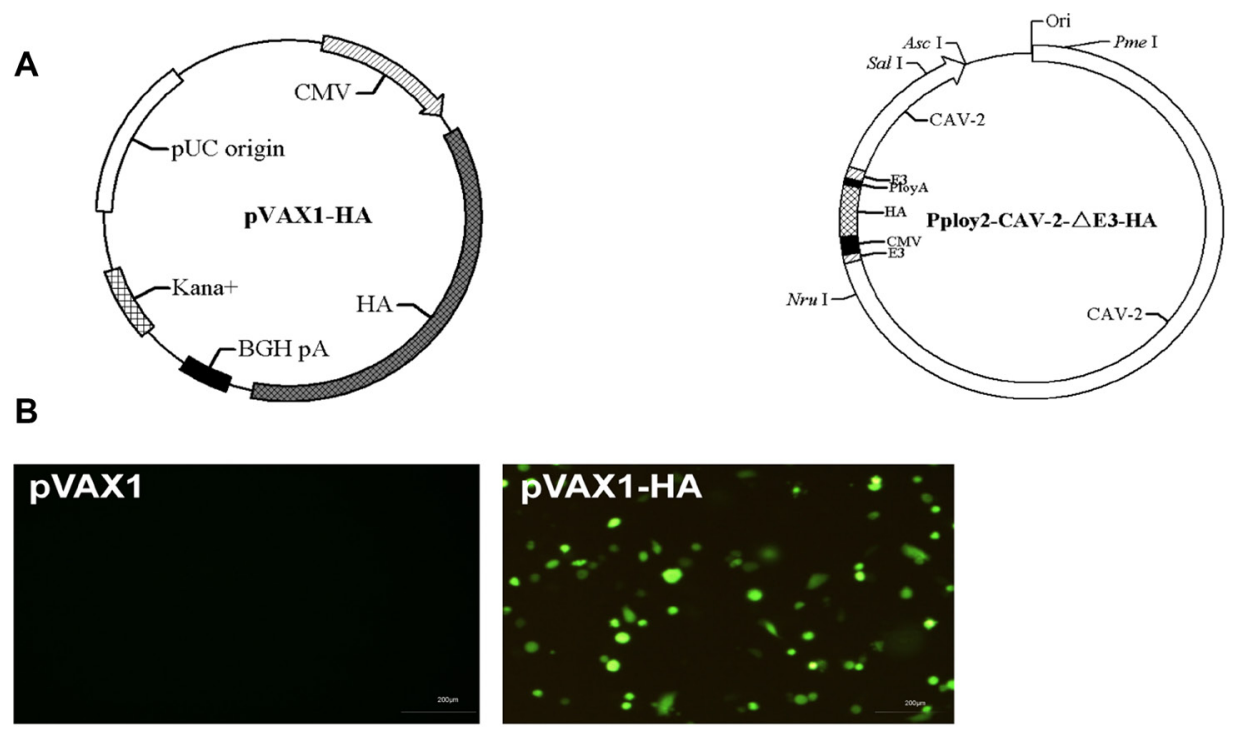

C
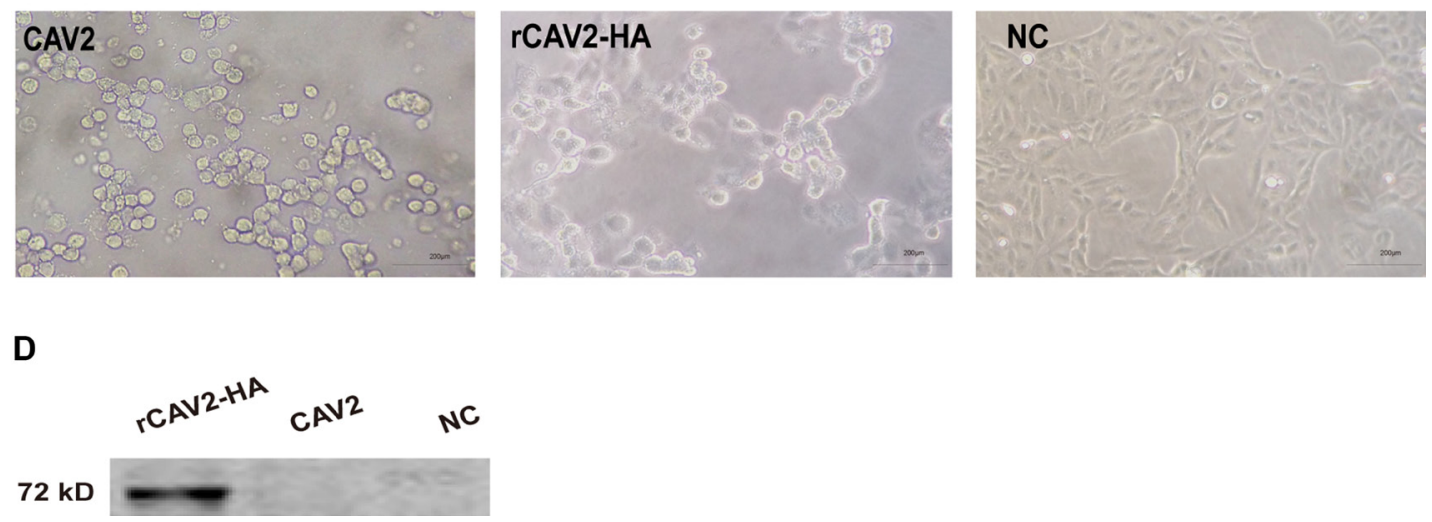

Figure 1: Construction and identification of the pVAX1-HA plasmid and recombined CAV2-HA. The hemagglutinin (HA) gene fragment of the canine influenza virus (A/canine/Guangdong/01/2006 (H3N2)) was inserted into the pVAX1 and ppoly2-CAV2- $\Delta$ E3 vectors (A). HA expression from the pVAX1-HA vector and rCAV2-HA was detected by indirect immunofluorescence and Western blot, respectively $(\mathbf{B}, \mathbf{D})$. In addition, MDCK cells exhibited grape-like gatherings indicative of an adenovirus infection after infection with rCAV2-HA for $24 \mathrm{~h}$ (C). 
Table 1: HI antibody titers were detected at 2, 4, 6 and $8 \mathrm{wpv}$

\begin{tabular}{cccccc}
\hline & & \multicolumn{3}{c}{ HI } \\
\cline { 3 - 6 } & & $\mathbf{2 ~ w p v}$ & $\mathbf{4}$ wpv. & $\mathbf{6}$ wpv & $\mathbf{8}$ wpv \\
\hline Group 1 & pVAX1-HA & 8 & 25.40 & $32^{\mathrm{a}, \mathrm{b}}$ & 12.70 \\
Group 2 & rCAV2-HA & 32 & 41.78 & $98.05^{\mathrm{a}, \mathrm{c}}$ & 25.40 \\
Group 3 & Inactivated vaccine & 32 & 47.95 & $120.82^{\mathrm{b}, \mathrm{c}}$ & 40.32 \\
Group 4 & pVAX1 & $<2$ & $<2$ & $<2$ & $<2$ \\
Group 5 & CAV2 & $<2$ & $<2$ & $<2$ & $<2$ \\
Group 6 & Adjuvant & $<2$ & $<2$ & $<2$ & $<2$ \\
Group 7 & PBS & $<2$ & $<2$ & $<2$ & $<2$ \\
Group 8 & NI & $<2$ & $<2$ & $<2$ & $<2$ \\
\hline
\end{tabular}

Notes:

Data are presented as geometric mean titer ( $n=12$, at 2, 4 and $6 \mathrm{wpv} ; n=3$, at $8 \mathrm{wpv})$.

Significant or extremely significant difference at $p<0.05$ or $p<0.01$, respectively.

$\mathrm{NI}=$ No immunization

$\mathrm{CAV} 2-\mathrm{HA}=$ live recombinant canine adenovirus $2(\mathrm{CAV} 2)$ vector expressing the $\mathrm{H} 3$ hemagglutinin protein from the virus strain A/canine/Guangdong/01/2006(H3N2).

CAV2 $=$ empty CAV2 vector

$\mathrm{wpv}=$ weeks post-vaccination

a,b,c The same letter indicates the intergroup difference analysis.

${ }^{a}$ The difference is extremely significant $(p<0.01)$; ${ }^{\mathrm{b}}$ The difference is extremely significant $(p<0.01)$; ${ }^{\mathrm{c}}$ The difference is not significant $(p>0.05)$.

cells [40]. Additionally, as a recombinant viral vector, CAV2 provides a safe and long-term protective effect in veterinary fields [41] that reduced the natural infection risk [42]. An inactivated viral-based vaccine is a traditional vaccine platform that is used to against a wide range of infections. Montanide ${ }^{\mathrm{TM}}$ Gel01 ST was used as adjuvants for both inactivated and live virus vaccines $[43,44]$ to enhance the protective efficacy in animals.

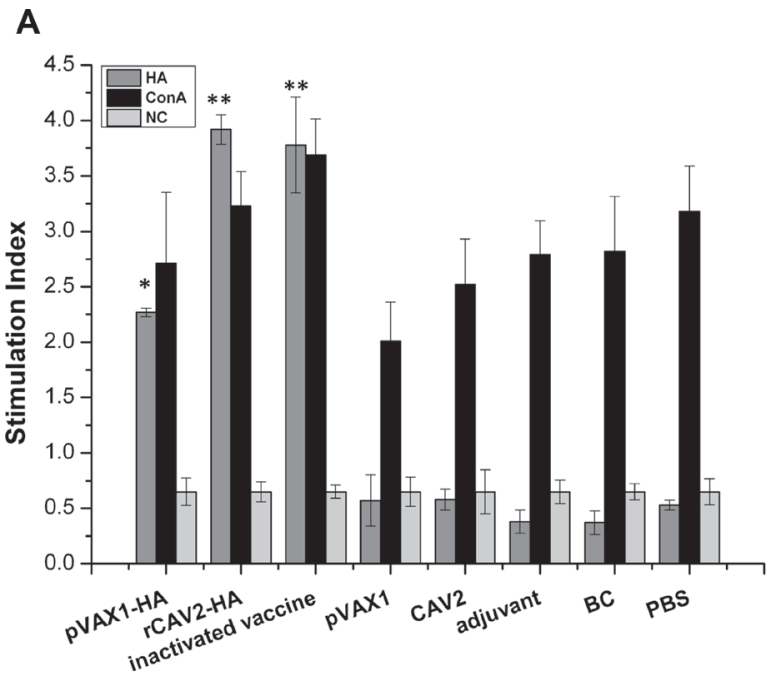

Of note, we observed that the HI titers of the rCAV2HA group were increased compared with the pVAX1-HA group but reduced compared with the inactivated group at the same time post vaccination (Table 1). This result reveals that the more CIV-specific antibodies for humoral immunity were produced by inactivated virus vaccination compared with other vaccines. The pVAX1-HA DNA group, which was exposed to a DNA vaccine, maintained

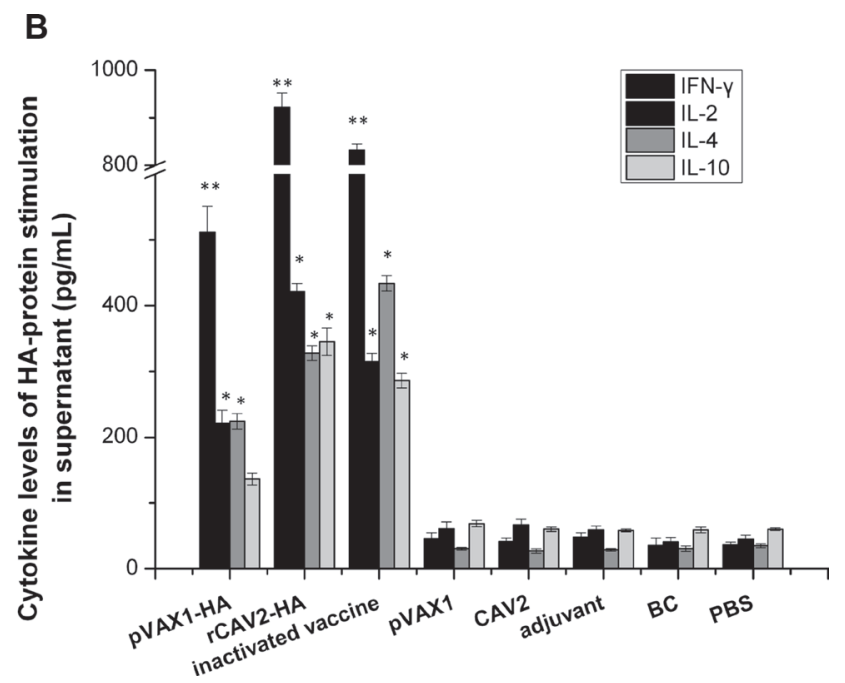

Figure 2: Splenic lymphocyte proliferation and cytokine secretion assays. At $6 \mathrm{wpv}$, the splenic lymphocytes from all mice $(n=3)$ were stimulated with antigen (HA protein and concanavalin A) after $72 \mathrm{~h}$. The SI was calculated as the ratio of the average $\mathrm{OD}_{450}$ value of wells containing antigen-stimulated cells to the mean $\mathrm{OD}_{450}$ value of wells containing only cells with medium (A). The secretion of cytokines against HA protein was measured via ELISA (B). Data are presented as the mean \pm SD. The difference is significant $\left({ }^{*} p<0.05\right)$ and extremely significant $\left({ }^{* *} p<0.01\right)$ compared with the related control group (inoculated mice with empty plasmid, adjuvant and PBS). 
an acceptable level of humoral immunity; however, the HI titers were reduced compare with the other two vaccine groups. The antibodies are effective against CIV infection and contribute to viral clearance in the organs.

IL- 2 and IFN- $\gamma$ are produced by Th1 cells to promote viral clearance by stimulating proliferation and cytotoxic T lymphocyte responses [45]. IL-4 is involved in a Th2 response, and IL-10 produces an anti-inflammatory response. Antigen-specific cellular responses were characterized based on the balance of Th2 and Th1 cytokines [46]. Cytokine secretion in the pVAX1-HA, rCAV2-HA and inactivated groups were increased, and the rCAV2-HA group exhibited the highest levels. This result suggests that the $\mathrm{T}$ cell-mediated immune response was activated, and live recombinant virus (rCAV2-HA group) stimulates an increased response compared with the inactivated virus vaccine (inactivated group) and the DNA vaccine (pVAX1-HA group). One explanation for this finding involves complete antigen epitope stimulation from live virus. In the splenic lymphocyte proliferation assay, the group with the highest SI increase was also the rCAV2-HA group given the persistent antigen presentation of live virus. This result implies that the lymphocytes specific to CIV HA protein were significantly responsive.
Histopathological lesions were detected in the respiratory tracts (trachea and lung) of CIV-challenged mice, which was consistent with the previous study [12]. We found that both the rCAV2-HA and the inactivated vaccine provided considerable protection against lung pathology and completely prevented weight loss following CIV challenge. However, some lung pathology and body weight loss were noted in mice receiving pVAX1-HA vaccination. However, the virus load was minimally detected after exposure to the three candidate vaccines. Previous research confirmed that H3N8 CIV (equine origin) resulted in an average loss of $5 \%$ body weight at 5 dpc [47].

Our results demonstrated that a live recombinant virus (rCAV2-HA) and an inactivated vaccine generated significant levels of immune activation in mice. Additionally, all three candidate vaccines displayed heightened protective efficacy against CIV H3N2 challenge, whereas limited protection was provided by the pVAX1-HA DNA vaccine. Therefore, both the rCAV2HA live recombinant virus and the inactivated CIV could be used as potential novel vaccines against the current epidemic of CIV H3N2. Moreover, the time of challenge with $\mathrm{H} 3 \mathrm{~N} 2 \mathrm{CIV}$ after vaccination was $2 \mathrm{wpv}$. If the time
A

\section{Lung (gross lesion)}

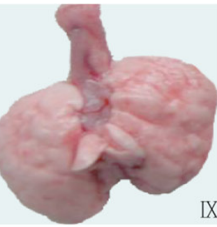

BC

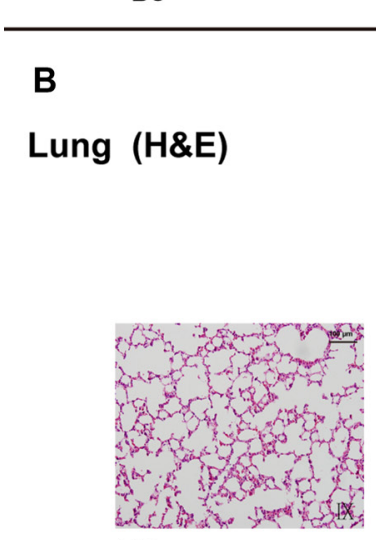

BC
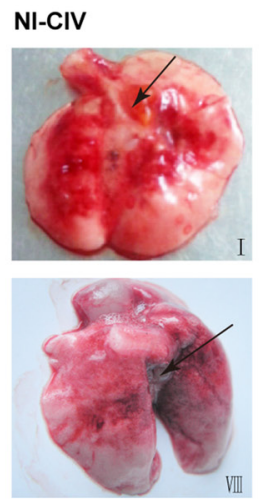

PBS-CIV
pVAX1-CIV
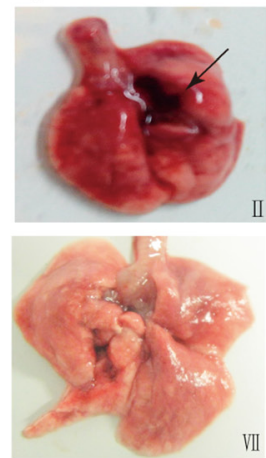

pVAX1-HA-CIV
CAV2-CIV

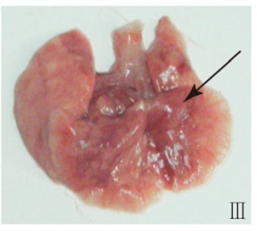

VII

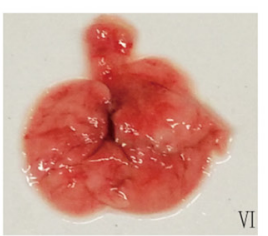

rCAV2-HA-CIV

\section{Adjuvant-CIV}
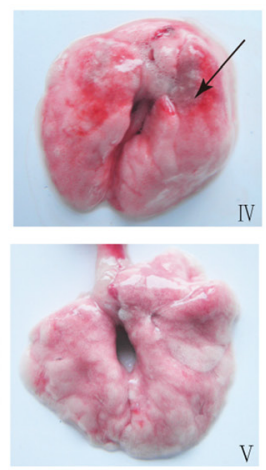

Inactivated CIV-CIV

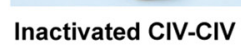

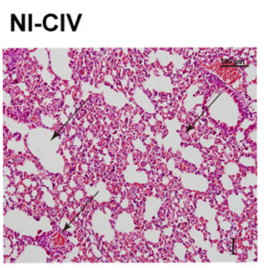
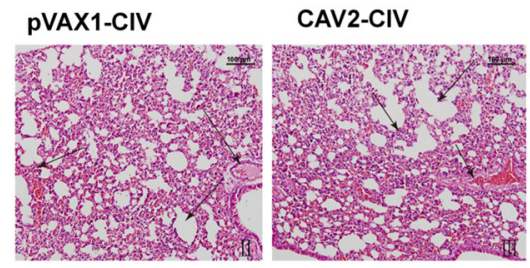

Adjuvant-CIV

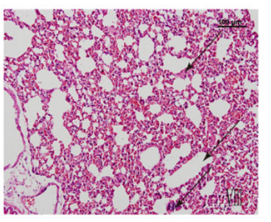

PBS-CIV

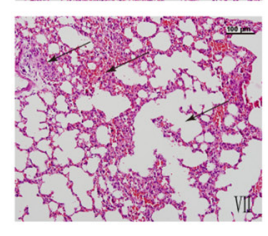

pVAX1-HA-CIV

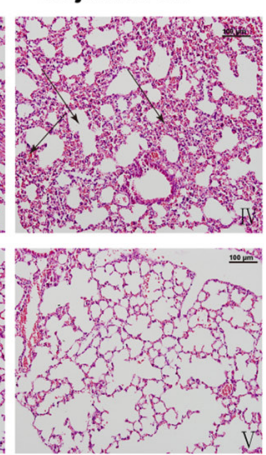

Inactivated CIV-CIV

Figure 3: Histopathology (200×). Lung lesions in mice at $5 \mathrm{dpc}$. No immunization and CIV challenge (I), pVAX1 vector vaccination and CIV challenge (II), CAV2 vaccination and CIV challenge (III), adjuvant inoculation and CIV challenge (IV), inactivated CIV vaccination and CIV challenge (V), rCAV2-HA vaccination and CIV challenge (VI), pVAX1-HA DNA vaccination and CIV challenge (VII), PBS inoculation and CIV challenge (VIII), and blank control (IX). 
of challenge was extended, a stronger immune response may be activated that would provide better protection from virus. In addition, a potential limitation should be noted. These CIV vaccines were only evaluated in mice, and the further studies in dogs should be explored.

\section{MATERIALS AND METHODS}

\section{Cells, virus and vectors}

MDCK cells (ATCC CRL-2936; American Type Culture Collection [ATCC], Manassas, VA) were maintained in Eagle's Minimum Essential Medium with 10\% fetal bovine serum (FBS) and an antibiotic solution $(10,000 \mathrm{IU} / \mathrm{mL}$ penicillin and $10,000 \mu \mathrm{g} / \mathrm{mL}$ streptomycin). All cells were incubated at $37^{\circ} \mathrm{C}$ and $5 \% \mathrm{CO}_{2}$. The avian-origin $\mathrm{CIV}$ strain $\mathrm{A} /$ canine/ Guangdong/01/2006 (H3N2) was used for the inactivated vaccine and challenge experiment [3]. The pVAX1 vector (Invitrogen, USA), a eukaryotic expression plasmid, was approved for recombined DNA vaccine and used according to the manufacturer's protocol [48]. The ppoly2CAV2- $\triangle \mathrm{E} 3$ vector is mainly composed of the partial E3 region of $\mathrm{CAV}-2$, human cytomegalovirus (hCMV) immediate-early gene promoter, polyA and the SV40 early mRNA polyadenylation signal. This vector was used for the recombined vaccine [49].

\section{Mice}

A total of 96 specific pathogen-free female BALB/c mice (six weeks old) were produced by the Center for Laboratory Animal Science of Guangdong and used for this study. All animal studies were conducted under guidelines approved by the Animal Care and Use Committee of the South China Agricultural University.

\section{Vaccines preparation}

The HA gene fragment amplified from the CIV genome was inserted into the pVAX1 vector to construct the recombinant plasmid pVAX1-HA, which was transferred into MDCK cells using Lipofectamine ${ }^{\circledR} 2000$ Transfection reagent (Invitrogen). The expression of HA mRNA and protein in MDCK cells were detected by RTPCR and indirect immunofluorescence assays (IFA). The pVAX1-HA plasmid used for vaccination was extracted from $E$. coli $\mathrm{DH} 5 \alpha$ using a no-endotoxin plasmid extract kit (TIANGEN, CHINA).

In addition, we generated the recombinant canine adenovirus type 2 virus (rCAV2-HA) expressing H3N2 CIV HA protein as previously described [49]. Briefly, the HA gene (A/canine/Guangdong/01/2006 (H3N2)) was inserted into the ppoly2-CAV2- $\triangle \mathrm{E} 3$ vector (ppoly2CAV2- $\triangle \mathrm{E} 3-\mathrm{HA})$. The chains and rings (1:1 ratio) were cotransferred into MDCK cells using Lipofectamine ${ }^{\circledR} 2000$ Transfection reagent, and the cells were cultivated at $37^{\circ} \mathrm{C}$ for $72 \mathrm{~h}$. Subsequently, the recombined ppoly2-CAV2$\triangle \mathrm{E} 3-\mathrm{HA}$ virus (rCAV2-HA) was collected and identified based on adenovirus cytopathologic characteristics as assessed by electron microscopy. HA protein expression in MDCK cells was verified by Western blotting, and the rabbit anti-H3N2 HA monoclonal antibody (obtained from our Lab) and IRDye 680RD goat (polyclonal) anti-rabbit IgG (LICOR, USA) were used. The viral titer of rCAV2HA and CIV were quantified by TCID $_{50}$ in MDCK cells as previously described $[50,51]$. The virus used for the vaccination experiment was used to inoculate MDCK cells
A

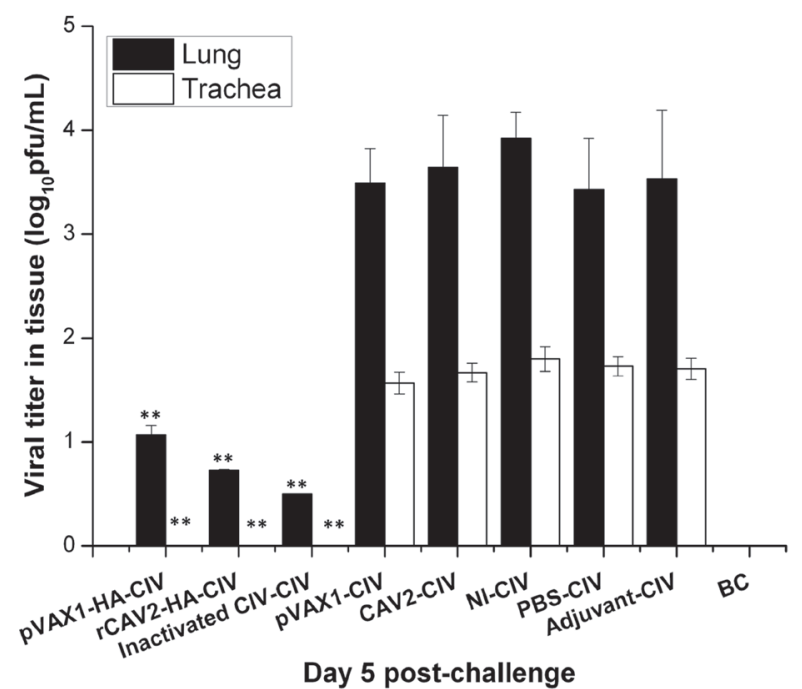

B

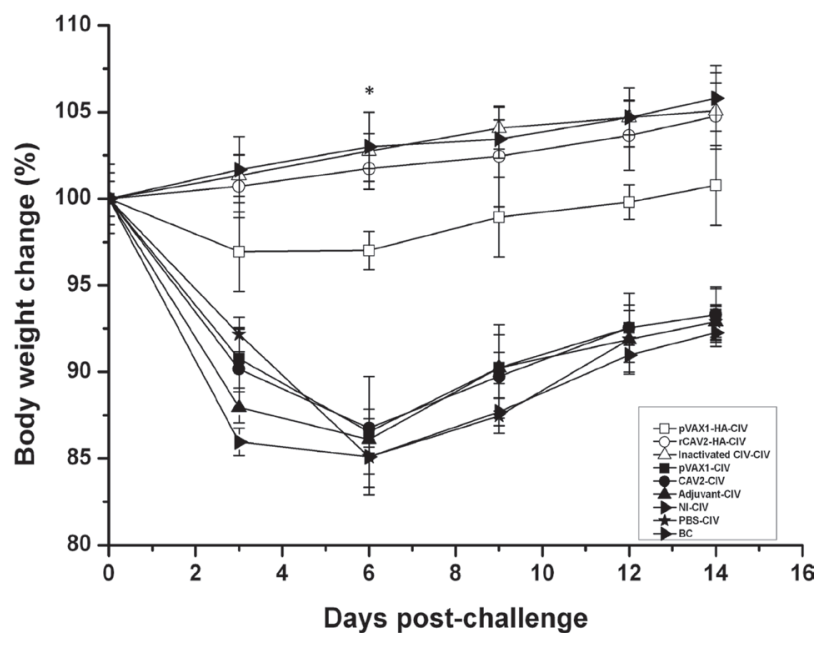

Figure 4: Virus load and change in body weight. Viral titers in the respiratory tract (lungs and tracheas) were detected using a plaque assay on $5 \mathrm{dpc}$ (A). On $0,3,6,9$, and $14 \mathrm{dpc}$, the body weight of mice was recorded, and the percent body weight change was calculated $(\mathbf{B})$. Data are presented as the mean $\pm \mathrm{SD}$ and analyzed as noted above. 
for viral amplification. The virus was purified by sucrose density gradient centrifugation before inactivation. The virus was inactivated with $0.05 \%$ formaldehyde [52] and assessed by performing serial passages in eggs according to the protocol published in European Pharmacopeia [53]. Inactivated CIV was inoculated in mice with an adjuvant, such as Montanide GEl 01st (SEPPIC, France), at a ratio of $80: 20$ by volume (according to the manufacturer's recommendations).

\section{Immunization}

The procedure for the immunization experiment is presented in Supplementary Figure 1. Mice were randomly divided into eight groups ( $n=12$ per group) and were intramuscularly injected with vaccines as follows: Group 1 (pVAX1-HA group), $100 \mu \mathrm{g}$ protein $\mathrm{pVAX} 1-\mathrm{HA}$ in $0.1 \mathrm{~mL}$; Group 2 (rCAV2-HA group), rCAV2-HA $\left(10^{4} \mathrm{TCID}_{50}\right)$ with $20 \mu \mathrm{L}$ adjuvant in $0.1 \mathrm{~mL}$; Group 3 (inactivated group), inactivated CIV $\left(10^{4} \mathrm{TCID}_{50}\right)$ with adjuvant in $0.1 \mathrm{~mL}$; Group 4 (pVAX1 group):, $100 \mu \mathrm{g}$ protein pVAX1 in $0.1 \mathrm{~mL}$; Group 5 (CAV2 group): CAV2 $\left(10^{4} \mathrm{TCID}_{50}\right)$ with $20 \mu \mathrm{L}$ adjuvant in $0.1 \mathrm{~mL}$; Group 6 (adjuvant group), $0.1 \mathrm{~mL}$ adjuvant; Group 7 (PBS group), $0.1 \mathrm{~mL}$ PBS; Group 8 (NI group), no immunization; Blank control group (BC), no immunization and no challenge.

Mice received an initial intramuscular immunization that was defined as $0 \mathrm{wpv}$. Then, mice received a booster immunization at 2 and $4 \mathrm{wpv}$. Mice were intramuscularly injected with an equal volume into each calf muscles.

\section{HI assay}

At two, four, six, and eight weeks after the initial vaccination, serum samples were collected from the tail vein of all mice to evaluate the $\mathrm{HI}$ antibody and cytokine expression levels. Sera were heat-inactivated (at $56^{\circ} \mathrm{C}$ for $30 \mathrm{~min}$ ) and treated with a receptor-destroying enzyme (RDE) (1:3) before the test. The HI assay was performed as described previously [54].

\section{Lymphocyte proliferation and cytokine production assay}

Briefly, splenocyte suspensions were isolated from mice (3/group) by pushing the spleens through a wire mesh one day prior to the challenge. RBCs were removed using the RBC lysis solution (Sigma), and splenocytes were supplemented with RPMI-1640 medium including $20 \% \mathrm{FBS}$ and an antibiotic solution $(10,000 \mathrm{IU} / \mathrm{mL}$ penicillin and $10,000 \mu \mathrm{g} / \mathrm{mL}$ streptomycin). Cells were plated in 96-well flat-bottom plates at $100 \mu \mathrm{L} /$ well $(5 \times$ $10^{5}$ cells/well). Subsequently, $100 \mu \mathrm{L} /$ well of medium containing HA protein $(10 \mu \mathrm{g} / \mathrm{mL})$, PBS (negative control) or Concanavalin A (ConA, $5 \mu \mathrm{g} / \mathrm{mL}$; Sigma) (positive control) was added at $37^{\circ} \mathrm{C}$ and $5 \% \mathrm{CO}_{2}$ for $72 \mathrm{~h}$. The proliferative activity was measured using a CCK8 kit (Beyotime, CHINA). The SI was calculated as the ratio of the average $\mathrm{OD}_{450}$ value of the wells containing antigenstimulated cells to the average $\mathrm{OD}_{450}$ value of the wells containing only cells with the medium. All assays were performed in triplicate. Passive secretion of cytokines (interleukin (IL)-2, IL-4, IL-10, and interferon (IFN)- $\gamma$ ) from collected supernatants was detected using specific ELISA kits (R\&D). Procedures were performed per the manufacturer's instructions. For each plate, the $\mathrm{OD}_{450}$ was transformed to a concentration by applying a linear regression formula, which was calculated from the standards of each kit.

\section{Challenge}

Mice ( $n=6$ /group, other 3 mice as blank control for each group) were anesthetized with isoflurane (3\%) and were intranasally inoculated with $0.1 \mathrm{~mL} \mathrm{CIV}\left(10^{5}\right.$ $\mathrm{TCID}_{50}$ ) at $6 \mathrm{wpv}$. The body weight was monitored and recorded on $0,3,6,9$, and $14 \mathrm{dpc}$. The rectal temperature was measured every dpc. On $5 \mathrm{dpc}, 3$ mice were euthanized by carbon dioxide inhalation in a gas chamber. After carbon dioxide treatment, heart rates, respiratory rates, and pupillary light reflexes were tested to confirm death. Lungs were collected rapidly and then immersed in $10 \%$ neutral formalin buffer overnight to prevent autolysis. The lungs were then sectioned and stained with HE for histopathological analysis. In addition, the virus titers in respiratory tract tissues were assessed by the plaque assay [55]. The remaining 3 mice were euthanized at $8 \mathrm{wpv}$.

\section{Statistical analysis}

All data were processed and analyzed using SPSS13.0 Data Editor (SPSS Inc., Chicago, IL, U.S.A.). Lymphoproliferation, cytokine production and viral titers were compared between groups using one-way ANOVA. Statistical analysis was performed using the unpaired Student's $t$-test. $p$ values of $p<0.05\left(^{*}\right)$ and $p<0.01$ $\left(^{* *}\right)$ were considered statistically significant and highly significant, respectively.

\section{Ethical}

All animal care and experimental procedures were performed under an approved protocol in compliance with CDC Institutional Animal Care and Use Guidelines.

\section{Abbreviations}

Canine influenza virus (CIV); United States of America (USA); Equine influenza virus (EIV); Phosphate buffered solution (PBS); Madin-Darby canine kidney (MDCK); Fetal bovine serum (FBS); Human cytomegalovirus (hCMV); Hemagglutination inhibition 
assay (HI); Weeks post-vaccination (wpv); Days postchallenge (dpc); Receptor-destroying enzyme (RDE); Red blood cells (RBC); Interferon (IFN); Interleukin (IL); Hematoxylin and eosin (HE); Stimulation index (SI).

\section{Author contributions}

Liqing $\mathrm{Tu}$, Lutao $\mathrm{Li}$, Xiuzhen $\mathrm{Li}$, Renjun $\mathrm{Hu}$ conducted the experiments. Liqing $\mathrm{Tu}$ and Pei Zhou analyzed the data and wrote the paper. Ziguo Yuan, Kun $\mathrm{Jia}$, and Lingshuang Sun revised the paper. Ziguo Yuan and Shoujun Li designed the experiments.

\section{CONFLICTS OF INTEREST}

The authors declare no conflicts of interest.

\section{FUNDING}

This project was supported in part by the National Natural Science Foundation of China (31672563, 31372448), The National Key Research and Development Program of China (2016YFD0501004), The Special Fund for Agroscientific Research in the Public Interest (201303042), The Promote Scientific and Technological Development Program (2013B040200032), and The Guangdong Provincial Key Laboratory of Prevention and Control for Severe Clinical Animal Diseases (2013A061401013).

\section{REFERENCES}

1. Crawford PC, Dubovi EJ, Castleman WL, Stephenson I, Gibbs EP, Chen L, Smith C, Hill RC, Ferro P, Pompey J, Bright RA, Medina MJ, Johnson CM, et al. Transmission of equine influenza virus to dogs. Science. 2005; 310:482-85.

2. Song D, Kang B, Lee C, Jung K, Ha G, Kang D, Park S, Park B, Oh J. Transmission of avian influenza virus (H3N2) to dogs. Emerg Infect Dis. 2008; 14:741-46.

3. Li S, Shi Z, Jiao P, Zhang G, Zhong Z, Tian W, Long LP, Cai Z, Zhu X, Liao M, Wan XF. Avian-origin H3N2 canine influenza A viruses in Southern China. Infect Genet Evol. 2010; 10:1286-88.

4. Centers for Disease Control and Prevention(CDC). Update on H3N2 Canine Influenza (Dog Flu) Virus. 2015. Available from: http://www.cdc.gov/flu/news/canine-influenza-sequencing.htm.

5. Watson CE, Bell C, Toohey-Kurth K. H3N2 Canine Influenza Virus Infection in a Dog. Vet Pathol. 2017; 54:527-30.

6. Zhou P, Huang S, Zeng W, Zhang X, Wang L, Fu X, Li S. Seroepidemiological evidence of subtype H3N8 influenza virus infection among pet dogs in China. PLoS One. 2016; 11:e0159106.

7. Newton R, Cooke A, Elton D, Bryant N, Rash A, Bowman $\mathrm{S}$, Blunden T, Miller J, Hammond TA, Camm I, Day M. Canine influenza virus: cross-species transmission from horses. Vet Rec. 2007; 161:142-43.
8. Kirkland PD, Finlaison DS, Crispe E, Hurt AC. Influenza virus transmission from horses to dogs, Australia. Emerg Infect Dis. 2010; 16:699-702.

9. Su S, Li HT, Zhao FR, Chen JD, Xie JX, Chen ZM, Huang Z, $\mathrm{Hu}$ YM, Zhang MZ, Tan LK, Zhang GH, Li SJ. Avian-origin H3N2 canine influenza virus circulating in farmed dogs in Guangdong, China. Infect Genet Evol. 2013; 14:444-49.

10. Yamanaka $T$, Nemoto $M$, Tsujimura $K$, Kondo $T$, Matsumura T. Interspecies transmission of equine influenza virus (H3N8) to dogs by close contact with experimentally infected horses. Vet Microbiol. 2009; 139:351-55.

11. Zhou H, He SY, Sun L, He H, Ji F, Sun Y, Jia K, Ning Z, Wang H, Yuan L, Zhou P, Zhang G, Li S. Serological evidence of avian influenza virus and canine influenza virus infections among stray cats in live poultry markets, China. Vet Microbiol. 2015; 175:369-73.

12. Lyoo KS, Na W, Yeom M, Jeong DG, Kim CU, Kim JK, Song D. Virulence of a novel reassortant canine H3N2 influenza virus in ferret, dog and mouse models. Arch Virol. $2016 ; 161: 1915-23$.

13. Lee YN, Lee DH, Park JK, Yuk SS, Kwon JH, Nahm SS, Lee JB, Park SY, Choi IS, Song CS. Experimental infection and natural contact exposure of ferrets with canine influenza virus (H3N2). J Gen Virol. 2013; 94:293-97.

14. Lyoo KS, Kim JK, Kang B, Moon H, Kim J, Song M, Park B, Kim SH, Webster RG, Song D. Comparative analysis of virulence of a novel, avian-origin H3N2 canine influenza virus in various host species. Virus Res. 2015; 195:135-40.

15. Dubovi EJ. Canine Influenza. Vet Clin N Am Small Anim Pract. 2010; 40:1063-71.

16. Dubovi EJ. Canine influenza. Vet Clin North Am Small Anim Pract. 2010; 40:1063-71.

17. Lee C, Song D, Kang B, Kang D, Yoo J, Jung K, Na G, Lee $\mathrm{K}$, Park B, Oh J. A serological survey of avian origin canine H3N2 influenza virus in dogs in Korea. Vet Microbiol. 2009; 137:359-62.

18. Sambhara V. Inactivated seasonal influenza vaccines increase serum antibodies to the neuraminidase of pandemic influenza A(H1N1) 2009 virus in an age-dependent manner. J Infect Dis. 2011; 203:1697-98.

19. Couch RB. Seasonal inactivated influenza virus vaccines. Vaccine. 2008 (Suppl 4); 26:D5-9.

20. $\mathrm{Xu} \mathrm{W}$, Zheng $\mathrm{M}$, Zhou $\mathrm{F}$, Chen Z. Long-term immunogenicity of an inactivated split-virion 2009 pandemic influenza A H1N1 virus vaccine with or without aluminum adjuvant in mice. Clin Vaccine Immunol. 2015; 22:327-35.

21. Zhao G, Lin Y, Du L, Guan J, Sun S, Sui H, Kou Z, Chan CC, Guo Y, Jiang S, Zheng BJ, Zhou Y. An M2e-based multiple antigenic peptide vaccine protects mice from lethal challenge with divergent H5N1 influenza viruses. Virol J. 2010; 7:9.

22. Ma JH, Yang FR, Yu H, Zhou YJ, Li GX, Huang M, Wen F, Tong G. An M2e-based synthetic peptide vaccine for 
influenza A virus confers heterosubtypic protection from lethal virus challenge. Virol J. 2013; 10:227.

23. Sui Z, Chen Q, Fang F, Zheng M, Chen Z. Cross-protection against influenza virus infection by intranasal administration of M1-based vaccine with chitosan as an adjuvant. Vaccine. 2010; 28:7690-98.

24. Maassab HF, Bryant ML. The development of live attenuated cold-adapted influenza virus vaccine for humans. Rev Med Virol. 1999; 9:237-44.

25. De Groot AS, Ardito M, McClaine EM, Moise L, Martin WD. Immunoinformatic comparison of T-cell epitopes contained in novel swine-origin influenza A (H1N1) virus with epitopes in 2008-2009 conventional influenza vaccine. Vaccine. 2009; 27:5740-47.

26. Mänz B, Götz V, Wunderlich K, Eisel J, Kirchmair J, Stech J, Stech O, Chase G, Frank R, Schwemmle M. Disruption of the viral polymerase complex assembly as a novel approach to attenuate influenza A virus. J Biol Chem. 2011; 286:8414-24.

27. Xie H, Liu TM, Lu X, Wu Z, Belser JA, Katz JM, Tumpey TM, Ye Z. A live attenuated H1N1 M1 mutant provides broad cross-protection against influenza $A$ viruses, including highly pathogenic A/Vietnam/1203/2004, in mice. J Infect Dis. 2009; 200:1874-83.

28. Esmagambetov IB, Sedova ES, Shcherbinin DN, Lysenko AA, Garas MN, Shmarov MM, Logunov DI. [Construction of recombinant adenoviral vector expressing genes of the conservative influenza proteins M2 and nucleoprotein]. [Article in Russian]. Mol Gen Mikrobiol Virusol. 2014; 2:22-28.

29. Kim EH, Park HJ, Han GY, Song MK, Pereboev A, Hong JS, Chang J, Byun YH, Seong BL, Nguyen HH. Intranasal adenovirus-vectored vaccine for induction of long-lasting humoral immunity-mediated broad protection against influenza in mice. J Virol. 2014; 88:9693-703.

30. Adam VS, Crosariol M, Kumar S, Ge MQ, Czack SE, Roy S, Haczku A, Tretiakova A, Wilson JM, Limberis MP. Adeno-associated virus 9-mediated airway expression of antibody protects old and immunodeficient mice against influenza virus. Clin Vaccine Immunol. 2014; 21:1528-33.

31. Pertmer TM, Oran AE, Moser JM, Madorin CA, Robinson HL. DNA vaccines for influenza virus: differential effects of maternal antibody on immune responses to hemagglutinin and nucleoprotein. J Virol. 2000; 74:7787-93.

32. Ogunremi O, Pasick J, Kobinger GP, Hannaman D, Berhane Y, Clavijo A, van Drunen Littel-van den Hurk S. A single electroporation delivery of a DNA vaccine containing the hemagglutinin gene of Asian H5N1 avian influenza virus generated a protective antibody response in chickens against a North American virus strain. Clin Vaccine Immunol. 2013; 20:491-500.

33. Flexner C, Moss B, London WT, Murphy BR. Attenuation and immunogenicity in primates of vaccinia virus recombinants expressing human interleukin-2. Vaccine. $1990 ; 8: 17-21$.
34. Karaca K, Dubovi EJ, Siger L, Robles A, Audonnet JC, Jiansheng Y, Nordgren R, Minke JM. Evaluation of the ability of canarypox-vectored equine influenza virus vaccines to induce humoral immune responses against canine influenza viruses in dogs. Am J Vet Res. 2007; 68:208-12.

35. Rosas C, Van de Walle GR, Metzger SM, Hoelzer K, Dubovi EJ, Kim SG, Parrish CR, Osterrieder N. Evaluation of a vectored equine herpesvirus type $1(\mathrm{EHV}-1)$ vaccine expressing $\mathrm{H} 3$ haemagglutinin in the protection of dogs against canine influenza. Vaccine. 2008; 26:2335-43.

36. Lee C, Jung K, Oh J, Oh T, Han S, Hwang J, Yeom M, Son D, Kim J, Park B, Moon H, Song D, Kang B. Protective efficacy and immunogenicity of an inactivated avian-origin H3N2 canine influenza vaccine in dogs challenged with the virulent virus. Vet Microbiol. 2010; 143:184-88.

37. Nobivac Flu Canine Bivalent (H3N2 + H3N8) Vaccine for Dogs $\mid$ Jeffers Pet. 2017.

38. Krammer F, Palese P, Steel J. Advances in universal influenza virus vaccine design and antibody mediated therapies based on conserved regions of the hemagglutinin. Curr Top Microbiol Immunol. 2015; 386:301-21.

39. Margine I, Krammer F. Animal models for influenza viruses: implications for universal vaccine development. Pathogens. 2014; 3:845-74.

40. Abdizadeh R, Maraghi S, Ghadiri AA, Tavalla M, Shojaee S. Cloning and Expression of Major Surface Antigen 1 Gene of Toxoplasma gondii RH Strain Using the Expression Vector pVAX1 in Chinese Hamster Ovary Cells. Jundishapur J Microbiol. 2015; 8:e22570.

41. Bangari DS, Mittal SK. Development of nonhuman adenoviruses as vaccine vectors. Vaccine. 2006; 24:849-62.

42. Fang Q, Shou-feng Z, Shu-chao W, Ye L, Jing-hui Z, Li-juan MI, Fei Z, Ying W, Rong-liang HU. Neutralizing antibody against canine adenovirus in dogs. Chin J Zoonoses. 2014; 30:155-57.

43. Li X, Galliher-Beckley A, Huang H, Sun X, Shi J. Peptide nanofiber hydrogel adjuvanted live virus vaccine enhances cross-protective immunity to porcine reproductive and respiratory syndrome virus. Vaccine. 2013; 31:4508-15.

44. Deville S, Arous JB, Bertrand FC, Borisov V, Dupuis L. Efficacy of intranasal and spray delivery of adjuvanted live vaccine against infectious bronchitis virus in experimentally infected poultry. Procedia Vaccinol. 2012; 6:85-92.

45. Wei H, Tan K, Sun R, Yin L, Zhang J, Pu Y. Aberrant production of Th1/Th2/Th17-related cytokines in serum of C57BL/6 mice after short-term formaldehyde exposure. Int J Environ Res Public Health. 2014; 11:10036-50.

46. Babiuk S, Skowronski DM, De Serres G, HayGlass K, Brunham RC, Babiuk L. Aggregate content influences the Thl/Th2 immune response to influenza vaccine: evidence from a mouse model. J Med Virol. 2004; 72:138-42.

47. Castleman WL, Powe JR, Crawford PC, Gibbs EP, Dubovi EJ, Donis RO, Hanshaw D. Canine H3N8 influenza virus infection in dogs and mice. Vet Pathol. 2010; 47:507-17. 
48. Farshadpour F, Makvandi M, Taherkhani R. Design, Construction and Cloning of Truncated ORF2 and tPAspPADRE-Truncated ORF2 Gene Cassette From Hepatitis E Virus in the pVAX1 Expression Vector. Jundishapur $\mathbf{J}$ Microbiol. 2015; 8:e26035.

49. Li XZ, Wang XH, Xia LJ, Weng YB, Hernandez JA, Tu LQ, Li LT, Li SJ, Yuan ZG. Protective efficacy of recombinant canine adenovirus type-2 expressing TgROP18 (CAV2-ROP18) against acute and chronic Toxoplasma gondii infection in mice. BMC Infect Dis. 2015; 15:114.

50. Cox RJ, Major D, Hauge S, Madhun AS, Brokstad KA, Kuhne M, Smith J, Vogel FR, Zambon M, Haaheim LR, Wood J. A cell-based H7N1 split influenza virion vaccine confers protection in mouse and ferret challenge models. Influenza Other Respi Viruses. 2009; 3:107-17.

51. Reed LJ, Muench HA. A simple method of estimating fifty percent endpoints. Am J Hyg. 1938; 27: 493-497.

52. Haredy AM, Takenaka N, Yamada H, Sakoda Y, Okamatsu M, Yamamoto N, Omasa T, Ohtake H, Mori Y, Kida H, Yamanishi K, Okamoto S. An MDCK cell culture-derived formalin-inactivated influenza virus whole-virion vaccine from an influenza virus library confers cross-protective immunity by intranasal administration in mice. Clin Vaccine Immunol. 2013; 20:998-1007.

53. European Pharmacopeia Influenza Vaccine. Split Virion, Inactivated. 2005; 01:158.

54. Jirjis FF, Deshpande MS, Tubbs AL, Jayappa H, Lakshmanan N, Wasmoen TL. Transmission of canine influenza virus (H3N8) among susceptible dogs. Vet Microbiol. 2010; 144:303-09.

55. Zhirnov OP, Ovcharenko AV, Bukrinskaya AG. A modified plaque assay method for accurate analysis of infectivity of influenza viruses with uncleaved hemagglutinin. Arch Virol. 1982; 71:177-83. 